\title{
Expression of neurones and neuronal precursors in the transition zone of short-segment Hirschsprung's disease
}

\author{
Authors: Jenna Doherty, ${ }^{A}$ Sumita Chhabra, ${ }^{A}$ Megan Smith, ${ }^{A}$ David Edgar, ${ }^{A}$ Sarah Almond, ${ }^{B}$ Simon Kenny ${ }^{B}$ and \\ Bettina Wilm ${ }^{A}$
}

\section{Introduction}

Hirschsprung's disease (HSCR) is a congenital disorder of dysfunctional enteric nervous system (ENS) development, resulting in distal aganglionosis of the bowel. ${ }^{1}$ The cellular composition of the transition zone (TZ), the region between the distal aganglionic bowel and normally innervated ganglionic bowel, is not well understood. Some children have resectional surgery in which TZ bowel is left in situ, with variable effects on functional outcome. ${ }^{2}$ Understanding the cellular anatomy of this region may aid in understanding the aetiology of HSCR and the effects of TZ pull-through on outcome, in addition to permitting the development of safe and effective use of ENS progenitor cells as therapy.

This work aimed to characterise the TZ of short-segment HSCR bowel, by assessing the expression of ENS and neuronal markers by immunofluorescence and quantitative polymerase chain reaction (qPCR).

\section{Materials and methods}

Human bowel was collected following a pull-through procedure in four patients with short-segment HSCR. Protein expression of ENS and neuronal markers (p75, SOX10, PHOX2b, Hu and GLUT1; Table 1) in ganglionic, proximal and distal TZ and aganglionic region of the bowel was visualised through immunofluorescence, and mRNA levels of the corresponding markers were quantified using qPCR.

\section{Results and discussion}

Immunofluorescence analysis showed a gradual loss of SOX10, $\mathrm{PHOX} 2 \mathrm{~b}$ and $\mathrm{Hu}$ protein in the lower $\mathrm{TZ}$ and absence in the aganglionic region. However, generally the expression of ENS and neuronal markers presented with inter-patient variability within the TZ. In contrast, GLUT1 was highly expressed in the perineurium of thickened nerve trunks, characteristic of the aganglionic region. Perineurial structures positive for GLUT1 were also visualised in the $\mathrm{TZ}$, but to a lesser extent than in

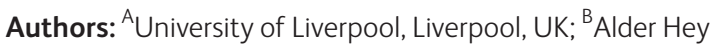
Children's Hospital, Liverpool, UK
Table 1. Neuronal markers studied in different

regions of the bowel

\begin{tabular}{ll}
$\begin{array}{l}\text { Neuronal/precursor } \\
\text { marker }\end{array}$ & Description \\
p75 & ENCC marker \\
GLUT1 & $\begin{array}{l}\text { Glucose transporter and expressed in } \\
\text { perineurium of nerves }\end{array}$ \\
Hu & Human neuronal marker \\
SOX10 & ENCC and glial cell marker \\
PHOX2b & ENCC and neural cell marker \\
\hline ENCC $=$ enteric neural crest cell &
\end{tabular}

ENCC $=$ enteric neural crest cell.

the aganglionic region. This observation corresponded with a decrease in mRNA levels of p75, SOX10, PHOX2b and $\mathrm{Hu}$ from the ganglionic to the aganglionic region and an increase in GLUT1.

While SOX10, PHOX2b and Hu positivity was not observed by immunofluorescence in the aganglionic region of bowel, mRNA of each marker was detected at low levels in each region via qPCR. Immunofluorescence visually detects protein expression, while qPCR quantifies mRNA levels. As mRNA is eventually transcribed into a protein, it may be assumed that there is a correlation between mRNA and protein levels, with qPCR and immunofluorescence results complementing each other. However, due to the many complicated and varied posttranscriptional mechanisms involved in protein production from $\mathrm{mRNA}$, there is potential for poor correlation between mRNA and protein expression, hence the potential for differing results between the methods. ${ }^{3}$

\section{Conclusion}

This work displayed a trend of a decreasing number of cells expressing ENS and neuronal markers from the ganglionic and aganglionic region, through the TZ. However, our analysis reveals inter-patient variability in the cellular composition of HSCR bowel, especially in the TZ. This finding may explain the variable functional outcome for HSCR children with TZ pull-through. 
Jenna Doherty, Sumita Chhabra, Megan Smith et al

\section{Conflicts of interest}

None declared.

\section{References}

1 Obermayr F, Hotta R, Enomoto H, Young HM. Development and developmental disorders of the enteric nervous system. Nat Rev Gastroenterol Hepatol 2013;10:43-57.
2 Engum SA, Grosfeld JL. Long-term results of treatment of Hirschsprung's disease. Semin Pediatr Surg 2004;13:273-85.

3 Greenbaum D, Colangelo C, Williams K, Gerstein M. Comparing protein abundance and mRNA expression levels on a genomic scale. Genome Biol 2003;4:117. 